\title{
The Influence of Passive-Dynamic Ankle-Foot Orthosis Bending Axis on Gait Performance in Individuals with Lower-Limb Impairments
}

\author{
Ellyn C. Ranz ${ }^{\mathrm{a}}$, Elizabeth Russell Esposito ${ }^{\mathrm{b}, \mathrm{c}}$, Jason M. Wilken ${ }^{\mathrm{b}, \mathrm{c}}$, Richard R. Neptune ${ }^{\mathrm{a}}$ \\ ${ }^{a}$ Department of Mechanical Engineering \\ The University of Texas at Austin \\ Austin, TX, 78712, USA \\ ${ }^{\mathrm{b}}$ Center for the Intrepid \\ Brooke Army Medical Center \\ JBSA Ft. Sam Houston, TX, 78234, USA \\ ${ }^{c}$ Extremity Trauma and Amputation Center of Excellence
}

Manuscript submitted to:

Clinical Biomechanics

October 2015

Address correspondence to: Richard R. Neptune

Department of Mechanical Engineering

The University of Texas at Austin

204 E. Dean Keeton Street, Stop C2200

Austin, TX, USA 78712-1591

Email: rneptune@mail.utexas.edu

Abstract Word Count: 249

Main Text Word Count: 4587 


\begin{abstract}
Background: Passive-dynamic ankle-foot orthoses are commonly prescribed to augment impaired ankle muscle function, however their design and prescription are largely qualitative. One design includes a footplate, cuff and flexible strut connecting the two. During gait, deflection occurs along the strut, with the greatest deflection at a central bending axis. The vertical location of the axis can affect lower extremity biomechanics. The goal of this study was to investigate the influence of bending axis location on gait performance.
\end{abstract}

Methods: For thirteen participants with unilateral ankle muscle weakness, an additive manufacturing framework was used to fabricate passive-dynamic ankle-foot orthosis struts with central and off-center bending axes. Participants walked overground while electromyographic, kinetic and kinematic data were collected for three different bending axes: proximal (high), central (middle) and distal (low), and the participants indicated their order of bending axis preference after testing. Gait measures and preference effect sizes were examined during six regions of the gait cycle.

Findings: The few differences between bending axes were observed in the first double-leg support peak plantarflexion angle, peak dorsiflexion moment and positive hip work, in the early single-leg support peak knee extension moment and positive ankle and knee work, and in the late single-leg support gastrocnemius activity and vertical ground reaction force impulse. In addition, preference was strongly related to various gait measures.

Interpretation: Despite the observed statistical differences, altering bending axis location did not produce large and consistent changes in gait performance. Thus, individual preference and comfort may be more important factors guiding prescription.

Keywords: PD-AFOs, limb salvage, selective laser sintering, gait, biomechanics 


\section{Introduction}

Recent military conflicts have resulted in a cohort of young, active individuals who have experienced traumatic injuries (Owens et al., 2007). Many injuries are the result of high energy blasts, frequently occurring at the foot or ankle (Tintle et al., 2010). As a result of surgical advancements, these injuries can often be treated with limb salvage procedures as opposed to amputation (Shawen et al., 2010). However, due to extensive musculoskeletal or neuromuscular injury, deficits in ankle strength and mobility may persist post-surgery and often an assistive device, such as an orthosis, is needed to help restore function. Passive-dynamic ankle-foot orthoses (PD-AFOs) are commonly prescribed to augment impaired ankle muscle function and restore walking ability in individuals with various lower-limb mobility impairments by providing elastic energy storage and return. Previous studies have demonstrated the beneficial effects of PD-AFOs on individuals with gait limitations (Desloovere et al., 2006; Patzkowski et al., 2012; Van Gestel et al., 2008), and have specifically examined the influence of PD-AFO stiffness on gait performance (Arch and Stanhope, 2015; Haight et al., 2015; Harper et al., 2014a; Russell Esposito et al., 2014) and energy cost (Bregman et al., 2011). However, the design and prescription process remains largely qualitative and modifications to PD-AFO design characteristics other than stiffness may be advantageous.

One PD-AFO design includes a molded footplate, cuff below the knee, and strut directly connecting the two (e.g., Fig.1a) (Patzkowski et al., 2011). During walking, deflection occurs along the length of the strut, with the location of greatest deflection or bending axis occurring at the center. Sumiya et al. (1997) observed that the AFO instantaneous center of rotation (representing the axis of a hingeless AFO as determined by AFO deformation) was located at the level of the ankle joint during plantarflexion movements and was located even more distally for 
dorsiflexion movements. Thus, PD-AFO bending axis is a design characteristic that may potentially influence ankle mechanics, yet it has been largely uninvestigated.

The alignment of the PD-AFO bending axis with the natural ankle joint may be beneficial for improving gait mechanics. Previous studies have examined the influence of AFO and physiological joint alignment in articulated AFOs, suggesting that alignment of the AFO joint with the natural ankle joint minimizes spatiotemporal and foot kinematic deviations from barefoot walking (Leardini et al., 2014) and results in minimal resistance torque (Gao et al., 2011). This finding has also been observed in articulated external fixations of the ankle (Bottlang et al., 1999). In addition, research has shown that misalignment of articulated AFOs with the ankle joint can lead to increases in cuff movement (Fatone and Hansen, 2007; Fatone et al., 2014), which may cause patient discomfort by generating skin and underlying tissue irritation on the shank.

To systematically study the effect of individual PD-AFO design characteristics, the ability to effectively replicate the features of clinical devices and manipulate design is required. To address this need an additive manufacturing framework was previously developed using selective laser sintering (SLS) (Harper et al., 2014b). SLS is an ideal additive manufacturing technique that can be used to efficiently fabricate customized, functional devices with precisely controlled design characteristics. SLS has previously been used to manufacture PD-AFOs (Faustini et al., 2008; Harper et al., 2014a; Schrank and Stanhope, 2011), as well as prosthetic sockets (Faustini et al., 2006; Rogers et al., 2007), feet (Fey et al., 2011; South et al., 2010) and ankles (Ventura et al., 2011a, b). In addition, PD-AFO components manufactured using SLS have been shown to influence gait performance in the same manner as clinically prescribed 
carbon fiber PD-AFOs (Harper et al., 2014b). Thus, SLS provides an ideal method to systematically alter PD-AFO bending axis.

Thus, the overall goal of this study was to use SLS to manufacture PD-AFOs with systematically varied bending axes and quantify the effect of the axis location on spatiotemporal, electromyographic, kinematic and kinetic measures. We hypothesize that subjects will prefer a more distal bending axis, which is located closer to the physiological ankle joint, and that this preference will correspond to improvements in biomechanical gait measures (closer to ablebodied). Insights gained by investigating the relationships between PD-AFO bending axis and gait performance will help identify the bending axis condition that results in the most normalized gait mechanics. This information can then be used to develop prescription guidelines for PDAFOs to enhance locomotor function and rehabilitation outcomes.

\section{Methods}

\subsection{Subjects}

A repeated-measures study design was used to compare across strut conditions. Thirteen participants who had experienced lower extremity trauma resulting in unilateral ankle muscle weakness and were undergoing rehabilitation at the Center for the Intrepid (Brooke Army Medical Center, JBSA Fort Sam Houston, TX) were enrolled in this study (Table 1). Each participant was clinically prescribed a PD-AFO (Intrepid Dynamic Exoskeletal Orthosis, Patzkowski et al., 2011). All participants provided institutionally-approved written informed consent prior to participation in this study and all data collection took place at the Military Performance Laboratory at the Center for the Intrepid. 


\subsection{SLS AFO Fabrication}

The clinically prescribed carbon fiber PD-AFO consisted of three components: a footplate, cuff and a strut connecting the footplate and cuff (Figure 1a). In this study, a strut emulating the prescribed carbon fiber strut stiffness with a central bending axis was manufactured for each subject using Nylon 11 (PD D80-ST, Advanced Laser Materials, LLC, Temple, TX, USA) with SLS (Vanguards HiQ/HS Sinterstation, 3D Systems, Inc., Rock Hill, SC, USA). A previously described SLS framework for designing and manufacturing the strut was used and summarized here (Harper et al., 2014b). The stiffness of the prescribed carbon fiber strut was determined through mechanical testing in a three-point-bend configuration with a support span of $160 \mathrm{~mm}$ and a maximum load of $890 \mathrm{~N}$ (5000 N uniaxial load cell, Instron, Norwood, MA, USA). Computer aided design (Solidworks, Dassault Systèmes Solidworks Corp., Waltham, MA, USA) and a predictive stiffness model were used to design each SLS strut. Manufactured SLS struts were subsequently tested in the same three-point-bend configuration to verify the stiffness matched the prescribed carbon fiber strut within 5\%.

The design and manufacture of an SLS strut possessing an off-center bending axis was performed using similar methods. The SLS strut design with a central bending axis was altered to offset the bending axis by decreasing the cross-sectional area of the strut, centered at $30 \%$ of the inner bolt hole-to-bolt hole distance from the center of the strut (Fig. 1b). The cross-sectional area of the strut was decreased using a $2.54 \mathrm{~cm}(1 \mathrm{inch})$ radius circular extrusion in the posterior half of the strut thickness. The cross-sectional area alteration was selected in order to bias the bending axis of the strut while minimizing stress concentrations. Finite element analyses were performed to adjust the altered SLS strut thickness to match the prescribed strut stiffness. Manufactured SLS struts were similarly tested in a three-point-bend configuration using a 
variable support span (the inner bolt hole-to-bolt hole distance) and a maximum load of $890 \mathrm{~N}$ (100 kN uniaxial load cell, MTS ReNew/Instron, Eden Prairie, MN, USA) to verify the stiffness. Adequate part strength and ductility were verified through the testing of SLS tensile specimens (5000 N uniaxial load cell, Instron, Norwood, MA, USA).

\subsection{Experimental Data Collection}

Participants performed overground walking trials while electromyographic (EMG), kinematic and kinetic data were collected. Participants were tested under three PD-AFO bending axis conditions: proximal bending axis (high), central bending axis (middle) and distal bending axis (low) (Fig. 1b-d). The SLS strut manufactured with an off-center bending axis was used for both the high and low bending axis conditions, and strut orientation determined the bending axis location. The order of device testing was randomized, and participants were given a minimum of 30 minutes acclimation time to each PD-AFO bending axis condition prior to testing. Flexible

lead tape (Clubmaker ${ }^{\mathrm{TM}}$, Golf Smith, Austin, TX, USA) was added along the length of each strut as needed to ensure equivalent PD-AFO masses across the bending axis conditions without altering strut bending stiffness.

For each of the bending axis conditions, participants performed overground walking at both their self-selected and Froude (0.16) (Vaughan and O'Malley, 2005) speeds. Surface EMG data (Motion Laboratory Systems, Inc., Baton Rouge, LA, USA) were collected at $1200 \mathrm{~Hz}$ bilaterally from seven muscles: soleus (SOL), medial gastrocnemius (GAS), tibialis anterior (TA), rectus femoris (RF), biceps femoris long head (BF), vastus medialis (VAS) and gluteus medius (GMED). Three-dimensional (3D) kinematics were collected at $120 \mathrm{~Hz}$ using a 26 camera motion capture system (Motion Analysis Corp., Santa Rosa, CA, USA) and a 6 degree- 
of-freedom body segment marker set with 57 markers (Wilken et al., 2012). Ground reaction forces (GRFs) were collected at $1200 \mathrm{~Hz}$ using force plates (AMTI, Inc., Watertown, MA, USA) embedded along the walkway. After all sessions were completed, participants indicated their ranked-order preference of the PD-AFO bending axis conditions.

\subsection{Data Processing}

\subsubsection{Electromyography}

Initial EMG processing was performed in Visual3D (C-Motion, Inc., Germantown, MD, USA). EMG data were demeaned, filtered using a bandpass filter (cutoff frequencies of $20 \mathrm{~Hz}$, $400 \mathrm{~Hz}$ ), smoothed with a $50 \mathrm{~ms}$ sliding RMS window and time-normalized to a full gait cycle. Five full gait cycles for both the PD-AFO and non-PD-AFO limb were exported for additional processing and analysis. In Matlab (Mathworks, Inc., Natick, MA, USA), EMG magnitudes were normalized by the peak value observed during the Froude speed condition for a given data collection session (Yang and Winter, 1984), and integrated EMG (iEMG) quantities were computed as the time integral of processed EMG data for each individual muscle. The iEMG quantities for each muscle were calculated within six regions of the gait cycle (Fig. 2): 1) first double-leg support, 2) early single-leg support, 3) late single-leg support, 4) second double-leg support, 5) early swing, and 6) late swing. The iEMG quantities were averaged across gait cycles and normalized by the full gait cycle iEMG for the middle bending axis condition.

\subsubsection{Kinematics and Kinetics}

In Visual3D, a 13-segment model was created and scaled by both participant mass and height. Model joint centers and coordinate systems were defined via 20 bilateral anatomical 
landmarks, defined through a digitization process in accordance with the International Society of Biomechanics standards (Grood and Suntay, 1983; Wu and Cavanagh, 1995; Wu et al., 2002). 3D kinematic and analog data were interpolated using a cubic polynomial and filtered with a low-pass, $4^{\text {th }}$-order Butterworth filter (cutoff frequencies of 6 and $50 \mathrm{~Hz}$, respectively). Euler angles were used to determine the joint kinematics and pelvis, hip, knee, and ankle kinematics were defined using Cardan rotation sequences (Baker, 2001; Grood and Suntay, 1983; Wu et al., 2002). Inverse dynamics were used to compute intersegmental joint moments and powers, which were normalized by subject mass, and GRF data were normalized by subject weight. GRF data in addition to joint kinematics, moments and powers were time normalized to a full gait cycle and five full gait cycles for each limb were exported for additional processing and analysis.

In subsequent processing and analysis in Matlab, changes in PD-AFO strut alignment between data collection sessions were normalized by subtracting the unloaded ankle angle during swing from the PD-AFO limb ankle angle across the full gait cycle. Peak joint kinematic and kinetic quantities were identified and GRF impulses and joint work were computed as the time integrals of GRFs and joint powers, respectively, during each of the six regions of the gait cycle (Fig. 2) and averaged across all gait cycles.

\subsection{Statistical Analyses}

To determine the influence of PD-AFO bending axis, differences in biomechanical measures were analyzed using two-way (bending axis condition, limb) repeated-measures ANOVAs using SPSS (IBM Corp, Armonk, NY, USA). Significant bending axis condition main effects and bending axis condition*limb interaction effects were adjusted using a Huynh-Feldt correction for sphericity violations and examined using post-hoc pairwise comparisons with a 
Bonferonni correction for multiple comparisons. The unadjusted criterion for statistical significance was set at $\mathrm{p}<0.05$. Significant pairwise comparisons between bending axis conditions for peak joint angles and moments were compared to previously-published minimal detectible change values calculated from data collected using identical methodology (Wilken et al., 2012).

In addition, a separate effect size analysis was conducted to assess the percent of variance in gait measures accounted for by participant PD-AFO bending axis preference (Cohen, 2013). Eta-squared calculations were performed using a general linear model in SPSS with preference as the fixed factor, and gait measures as the dependent variables. The measure of association was considered to be large for $\eta^{2}>0.26$, and values of $\eta^{2}>0.50$ were additionally noted to highlight gait measures for which over half of the variance can be accounted for by preference.

\section{Results}

Of the thirteen participants, four participants preferred the low bending axis, seven preferred the middle bending axis, and two preferred the high bending axis. The average walking speed was $1.32(\mathrm{SD} 0.07) \mathrm{m} / \mathrm{s}$ for their self-selected speed, and $1.25(\mathrm{SD} 0.05) \mathrm{m} / \mathrm{s}$ for their Froude speed. Similar trends across all variables were observed at their self-selected and Froude speeds, and thus the results for the Froude speed are included as Supplemental Material and the results for the self-selected walking speed are presented below. Further, there were no significant differences in walking speed, stride length and stride width across the bending axis conditions at either the self-selected or Froude speed.

\section{$3.1 \mathrm{iEMG}$}


In Region 3, the bending axis location influenced GAS (bending axis main effect, $\mathrm{p}=$ 0.040; bending axis*limb interaction effect, $\mathrm{p}=0.043$ ) and TA (bending axis*limb interaction effect, $p=0.047$ ) activity (Fig. 3). For GAS, iEMG values were greater in the low compared to the middle condition $(15.4 \%, \mathrm{p}=0.030)$, particularly in the PD-AFO limb $(30.9 \%, \mathrm{p}=0.009)$.

Large participant bending axis preference effect sizes $\left(\eta^{2}>0.26\right)$ were observed for iEMG values for all recorded muscles (Fig. 3). During Region 1 in the PD-AFO limb, preference accounted for over half of the variance in the TA iEMG for the low condition $\left(\eta^{2}=0.62\right)$, while in the non-PD-AFO limb, preference accounted for over half of the variance in the BF $\left(\eta^{2}=0.61\right)$ and SOL $\left(\eta^{2}=0.58\right)$ iEMG for the low condition and in the GAS iEMG for the high condition $\left(\eta^{2}=0.55\right)$. In Region 4, preference accounted for over half of the variance in the PD-AFO limb TA iEMG for the low condition $\left(\eta^{2}=0.64\right)$. In Region 5 , preference accounted for over half of the variance in the PD-AFO limb SOL iEMG for the high condition $\left(\eta^{2}=0.64\right)$ and in the nonPD-AFO limb VAS iEMG for the high condition $\left(\eta^{2}=0.52\right)$. In Region 6 , preference accounted for over half of the variance in the PD-AFO limb VAS $\left(\eta^{2}=0.59\right)$ and SOL $\left(\eta^{2}=0.58\right)$ iEMG for the high condition.

\subsection{Joint Kinematics and Kinetics}

Peak plantarflexion angle (Region 1: bending axis main effect, $\mathrm{p}=0.002$ ), dorsiflexion moment (Region 1: bending axis main effect, $\mathrm{p}=0.006$ ) and knee extension moment (Region 2: bending axis main effect, $\mathrm{p}=0.010$ ) were influenced by PD-AFO bending axis condition (Table 2). In Region 1, the initial plantarflexion angle and dorsiflexion moment were greater in the low compared to the middle condition (angle: $24.3 \%, \mathrm{p}=0.004$; moment: $12.9 \%, \mathrm{p}=0.019$ ). In Region 2, the peak knee extension moment was lower in the middle compared to the high 
condition $(11.9 \%, \mathrm{p}=0.039)$. However, each of these significant pairwise comparisons (i.e., Region 1 peak plantarflexion angle and dorsiflexion moment, and Region 2 peak knee extension moment) displayed differences that were less than previously-published minimal detectible change values for self-selected walking speeds (Wilken et al., 2012).

Large participant bending axis preference effect sizes $\left(\eta^{2}>0.26\right)$ were observed for peak joint kinematics and kinetics at the ankle, knee and hip (Table 2). Preference accounted for over half of the variance in the Region 4 PD-AFO limb peak dorsiflexion angle with the low condition $\left(\eta^{2}=0.52\right)$, and peak plantarflexion moment with the low $\left(\eta^{2}=0.86\right)$, middle $\left(\eta^{2}=0.60\right)$, and high $\left(\eta^{2}=0.67\right)$ conditions. Preference accounted for over half of the variance in the Region 2 non-PD-AFO limb knee flexion angle with the middle condition $\left(\eta^{2}=0.55\right)$ and Region 5 nonPD-AFO limb knee flexion angle with the high condition $\left(\eta^{2}=0.56\right)$.

\subsection{Joint Work}

PD-AFO bending axis influenced joint work at the ankle (Region 1 negative work: bending axis main effect, $\mathrm{p}=0.016$; Region 2 positive work: bending axis main effect, $\mathrm{p}=$ 0.003), knee (Region 2 positive work: bending axis main effect, $\mathrm{p}=0.011$ ) and hip (Region 1 positive work: bending axis main effect, $\mathrm{p}=0.008$; Region 3 negative work; bending axis main effect, $\mathrm{p}=0.046$ ) (Fig. 4). In Region 1, positive hip work was $20.8 \%$ greater for the middle compared to the high condition $(\mathrm{p}=0.011)$. During Region 2, positive ankle work was $34.1 \%$ lower for the middle compared to the low condition $(\mathrm{p}=0.002)$ and positive knee work was $24.7 \%$ lower for the middle compared to the high condition $(\mathrm{p}=0.027)$.

Large participant bending axis preference effect sizes $\left(\eta^{2}>0.26\right)$ were observed in joint work at the ankle, knee and hip (Fig. 4). In Region 2, preference accounted for over half of the variance in the non-PD-AFO limb positive hip work for the middle condition $\left(\eta^{2}=0.57\right)$. In 
Region 3, preference accounted for over half of the variance in the PD-AFO limb negative knee work for the high condition $\left(\eta^{2}=0.58\right)$. In Region 4 , preference accounted for over half of the variance in the PD-AFO limb positive ankle work for the low condition $\left(\eta^{2}=0.59\right)$ and positive hip work for the high condition $\left(\eta^{2}=0.51\right)$. In Region 6 , preference accounted for over half of the variance in the PD-AFO limb positive hip work for the high condition $\left(\eta^{2}=0.54\right)$ and nonPD-AFO limb positive hip work for the low $\left(\eta^{2}=0.52\right)$ and high $\left(\eta^{2}=0.60\right)$ conditions.

\subsection{GRF Impulses}

The only significant difference in GRF impulses across bending axis conditions occurred in the vertical GRF impulse (Region 3: bending axis main effect, $\mathrm{p}=0.011$ ) (Fig. 5). In Region 3 , the vertical GRF impulse with the middle condition was greater than both the low $(3.2 \%, \mathrm{p}=$ $0.011)$ and high $(1.9 \%, \mathrm{p}=0.003)$ conditions.

Large participant bending axis preference effect sizes $\left(\eta^{2}>0.26\right)$ were observed in the anteroposterior, vertical and mediolateral GRF impulses (Fig. 5). Preference accounted for over half of the variance in the vertical GRF impulse during Region 1 for the non-PD-AFO limb with the high condition $\left(\eta^{2}=0.57\right)$, as well as during Region 3 for the PD-AFO limb with the middle condition $\left(\eta^{2}=0.66\right)$.

\section{Discussion}

The goal of this study was to investigate the influence of PD-AFO bending axis on gait performance. A better understanding of the relationship between bending axis and gait performance would help facilitate the development of evidence-based prescription guidelines for PD-AFOs and ultimately, improve rehabilitation outcomes for PD-AFO users. 
PD-AFO bending axis altered GAS activity (iEMG) in late single-leg support. The low bending axis resulted in the greatest GAS activity, which has been previously shown to be an important contributor to both body support and forward propulsion in single-leg stance (Liu et al., 2006; Neptune et al., 2001). Harper et al. (2014a) similarly observed changes in GAS activity as a result of changes in PD-AFO stiffness in similar limb salvage subjects.

Altering PD-AFO bending axis had few effects on peak joint kinematics and kinetics. In first double-leg support, the peak plantarflexion angle and peak dorsiflexion moment were greater in the low condition, and in early single-leg support, the knee extension moment was greater in the high condition. Previous studies have observed a relationship between the instantaneous center of rotation of hingeless AFOs and ankle joint mechanics (Sumiya et al., 1997), as well as suggested that alignment of an articulated AFO joint with the physiological ankle joint minimizes foot kinematic deviations from barefoot walking (Leardini et al., 2014). This suggests that the use of the low bending axis, which lies closest to the physiological ankle joint, may allow for more normalized gait kinematics. However, the findings of the current study were not in complete agreement, as the shift away from values previously observed in unimpaired subjects (Russell Esposito et al., 2014) was seen in both the high and low conditions (i.e., the increased knee extension moment observed in early single-leg support in the high condition as well as the increased peak dorsiflexion moment observed during first double-leg support in the low condition). It is important to note, however, that while significant peak joint kinematic and kinetic differences were observed in the current study, these values were lower than previously published minimal detectable change values for level walking at self-selected speeds (Wilken et al., 2012), and thus are likely not clinically relevant. 
In the first half of stance, positive and negative ankle work, positive knee work and positive hip work were influenced by PD-AFO bending axis condition. In the second half of stance, negative hip work was influenced by PD-AFO bending axis condition. However, it should be noted that a consistent trend (e.g., increasing values as bending axis location moved from distal to proximal) was not observed.

Contrary to our hypothesis, the majority of participants preferred the middle bending axis, although at least two participants preferred each of the other bending axis locations. Inconsistent preferences in PD-AFO design between limb salvage subjects has also been observed when investigating PD-AFO stiffness in both level (Russell Esposito et al., 2014) and sloped walking (Haight et al., 2015). In addition, Raschke et al. (2015) suggested that there may be a link between prosthetic foot design preference and gait biomechanics, with individuals tending to prefer a prosthetic foot stiffness that minimized peak sagittal plane joint moments. The results of the current study also suggest a strong relationship between bending axis preference and peak sagittal plane joint moments. A post-hoc analysis indicated that minimization of PD-AFO limb peak hip flexion moment and ankle plantarflexion moment during second double-leg support as well as the non-PD-AFO limb peak ankle dorsiflexion moment during first double-leg support and peak knee extension moment during early single-leg support each occurred in the preferred bending axis condition for over half of the participants. In addition to peak joint moments, peak joint kinematics, joint work, GRF impulses and muscle activity were also strongly related to bending axis preference. The differences in participant preference may have been influenced by diversity in etiology, and future work with larger preference subgroups and additional functional capability testing (e.g., range of motion), may prove beneficial to addressing the relationship between etiology and preferred PD-AFO prescription. 
In this study, there are a few limitations that warrant discussion. First, the participants enrolled in this study had a diverse range of injuries that led to the prescription of a PD-AFO. It is possible that etiology may be a confounding factor in the influence of PD-AFO bending axis on gait performance. The range of injuries and resulting functional limitations were diverse in this cohort and each participant's bending axis preference may have been directly related to their individual etiology. Although many of the participants had similar clinical presentations with respect to functional deficits, given the heterogeneity of injuries, very large cohorts of participants would likely be required to fully account for the role of etiology. By examining the relationships between preference and biomechanical measures through an effect size analysis, our goal was to indicate the presence of interactions between etiology and the influence of PDAFO bending axis on gait. Also, the participants enrolled in this study were young, highly active individuals and may not be representative of other populations of AFO and PD-AFO users. Therefore, future work examining the influence of PD-AFO bending axis in additional populations would be beneficial.

Finally, the contribution of the PD-AFO to ankle joint moments and work could not be distinguished from physiological contributions in this experimental setup. Previous studies have shown that alignment of an articulated AFO joint (Gao et al., 2011) and an articulated external ankle fixation joint (Bottlang et al., 1999) with the physiological ankle joint results in minimal resistance torque. Thus, it is likely that PD-AFO contributions to net ankle joint moments and work were influenced by bending axis location. Therefore, future work quantifying this contribution would improve the understanding of the influence of PD-AFO bending axis on compensatory mechanisms. 


\section{Conclusions}

In conclusion, several significant differences were observed between the specified bending axis conditions, including the peak plantarflexion angle, peak dorsiflexion moment and positive hip work during first double-leg support, peak knee extension moment and positive ankle and knee work during early single-leg support, and GAS activity and vertical GRF impulse during late single-leg support. Although these differences were observed, peak joint kinematic and kinetic differences were less than previously published minimal detectable changes, and moving the bending axis proximally or distally did not produce large and consistent changes in other gait measures. This suggests that choice of bending axis does not significantly change gait and other untested factors, such as etiology, may play a role in level ground walking mechanics. As very large cohorts of participants would be required to further evaluate the role of etiology, at present individual preference and comfort may be more important factors guiding the prescription of PD-AFO bending axis for level ground walking.

\section{Acknowledgements}

The authors would like to thank the members of the Military Performance Laboratory at the Center for the Intrepid for their contributions to this study in participant recruitment, experimental data collection and data processing. This study was supported in part by a research grant from the Center for Rehabilitation Sciences Research (CRSR), Department of Physical Medicine and Rehabilitation, Uniformed Services University of Health Sciences, Bethesda, MD. The views expressed herein are those of the authors and do not reflect the official policy or position of Brooke Army Medical Center, the U.S. Army Medical Department, the U.S. Army 
Office of the Surgeon General, the Department of the Army, Department of Defense or the U.S.

Government. 


\section{References}

Arch, E., Stanhope, S., 2015. Passive-dynamic ankle-foot orthoses substitute for ankle strength while causing adaptive gait strategies: a feasibility study. Annals of Biomedical Engineering 43, 442-450.

Baker, R., 2001. Pelvic angles: a mathematically rigorous definition which is consistent with a conventional clinical understanding of the terms. Gait Posture 13, 1-6.

Bottlang, M., Marsh, J.L., Brown, T.D., 1999. Articulated external fixation of the ankle: minimizing motion resistance by accurate axis alignment. J Biomech 32, 63-70.

Bregman, D.J., van der Krogt, M.M., de Groot, V., Harlaar, J., Wisse, M., Collins, S.H., 2011. The effect of ankle foot orthosis stiffness on the energy cost of walking: a simulation study. Clin Biomech 26, 955-961.

Cohen, J., 2013. The analysis of variance, Statistical power analysis for the behavioral sciences, 2 ed. Taylor and Francis, pp. 274-288.

Desloovere, K., Molenaers, G., Van Gestel, L., Huenaerts, C., Van Campenhout, A., Callewaert, B., Van de Walle, P., Seyler, J., 2006. How can push-off be preserved during use of an ankle foot orthosis in children with hemiplegia? A prospective controlled study. Gait Posture 24, 142-151.

Fatone, S., Hansen, A.H., 2007. A model to predict the effect of ankle joint misalignment on calf band movement in ankle-foot orthoses. Prosthet Orthot Int 31, 76-87.

Fatone, S., Johnson, W.B., Kwak, S., 2014. Using a three-dimensional model of the ankle-foot orthosis/leg to explore the effects of combinations of axis misalignments. Prosthet Orthot Int (in press).

Faustini, M.C., Neptune, R.R., Crawford, R.H., Rogers, W.E., Bosker, G., 2006. An experimental and theoretical framework for manufacturing prosthetic sockets for transtibial amputees. IEEE Trans Neural Syst Rehabil Eng 14, 304-310.

Faustini, M.C., Neptune, R.R., Crawford, R.H., Stanhope, S.J., 2008. Manufacture of passive dynamic ankle-foot orthoses using selective laser sintering. IEEE Trans Biomed Eng 55, 784790 .

Fey, N.P., Klute, G.K., Neptune, R.R., 2011. The influence of energy storage and return foot stiffness on walking mechanics and muscle activity in below-knee amputees. Clin Biomech 26, 1025-1032.

Gao, F., Carlton, W., Kapp, S., 2011. Effects of joint alignment and type on mechanical properties of thermoplastic articulated ankle-foot orthosis. Prosthet Orthot Int 35, 181-189.

Grood, E.S., Suntay, W.J., 1983. A joint coordinate system for the clinical description of threedimensional motions: application to the knee. J Biomech Eng 105, 136-144. 
Haight, D.J., Russell Esposito, E., Wilken, J.M., 2015. Biomechanics of uphill walking using custom ankle-foot orthoses of three different stiffnesses. Gait Posture 41, 750-756.

Harper, N.G., Esposito, E.R., Wilken, J.M., Neptune, R.R., 2014a. The influence of ankle-foot orthosis stiffness on walking performance in individuals with lower-limb impairments. Clin Biomech 29, 877-884.

Harper, N.G., Russell, E.M., Wilken, J.M., Neptune, R.R., 2014b. Selective laser sintered versus carbon fiber passive-dynamic ankle-foot orthoses: a comparison of patient walking performance. J Biomech Eng 136, 091001.

Leardini, A., Aquila, A., Caravaggi, P., Ferraresi, C., Giannini, S., 2014. Multi-segment foot mobility in a hinged ankle-foot orthosis: the effect of rotation axis position. Gait Posture 40, 274277.

Liu, M.Q., Anderson, F.C., Pandy, M.G., Delp, S.L., 2006. Muscles that support the body also modulate forward progression during walking. J Biomech 39, 2623-2630.

Neptune, R.R., Kautz, S.A., Zajac, F.E., 2001. Contributions of the individual ankle plantar flexors to support, forward progression and swing initiation during walking. J Biomech 34, 13871398.

Owens, B.D., Kragh, J.F., Jr., Macaitis, J., Svoboda, S.J., Wenke, J.C., 2007. Characterization of extremity wounds in Operation Iraqi Freedom and Operation Enduring Freedom. J Orthop Trauma 21, 254-257.

Patzkowski, J.C., Blanck, R.V., Owens, J.G., Wilken, J.M., Blair, J.A., Hsu, J.R., 2011. Can an ankle-foot orthosis change hearts and minds? J Surg Orthop Adv 20, 8-18.

Patzkowski, J.C., Blanck, R.V., Owens, J.G., Wilken, J.M., Kirk, K.L., Wenke, J.C., Hsu, J.R., 2012. Comparative effect of orthosis design on functional performance. J Bone Joint Surg Am 94, 507-515.

Raschke, S.U., Orendurff, M.S., Mattie, J.L., Kenyon, D.E., Jones, O.Y., Moe, D., Winder, L., Wong, A.S., Moreno-Hernandez, A., Highsmith, M.J., D, J.S., Kobayashi, T., 2015. Biomechanical characteristics, patient preference and activity level with different prosthetic feet: a randomized double blind trial with laboratory and community testing. J Biomech 48, 146-152.

Rogers, B., Bosker, G.W., Crawford, R.H., Faustini, M.C., Neptune, R.R., Walden, G., Gitter, A.J., 2007. Advanced trans-tibial socket fabrication using selective laser sintering. Prosthet Orthot Int 31, 88-100.

Russell Esposito, E., Blanck, R.V., Harper, N.G., Hsu, J.R., Wilken, J.M., 2014. How does ankle-foot orthosis stiffness affect gait in patients with lower limb salvage? Clin Orthop Relat Res 472, 3026-3035.

Schrank, E.S., Stanhope, S.J., 2011. Dimensional accuracy of ankle-foot orthoses constructed by rapid customization and manufacturing framework. J Rehabil Res Dev 48, 31-42. 
Shawen, S.B., Keeling, J.J., Branstetter, J., Kirk, K.L., Ficke, J.R., 2010. The mangled foot and leg: salvage versus amputation. Foot Ankle Clin 15, 63-75.

South, B.J., Fey, N.P., Bosker, G., Neptune, R.R., 2010. Manufacture of energy storage and return prosthetic feet using selective laser sintering. J Biomech Eng 132, 015001.

Sumiya, T., Suzuki, Y., Kasahara, T., Ogata, H., 1997. Instantaneous centers of rotation in dorsi/plantar flexion movements of posterior-type plastic ankle-foot orthoses. J Rehabil Res Dev $34,279-285$.

Tintle, S.M., Keeling, J.J., Shawen, S.B., 2010. Combat foot and ankle trauma. J Surg Orthop Adv 19, 70-76.

Van Gestel, L., Molenaers, G., Huenaerts, C., Seyler, J., Desloovere, K., 2008. Effect of dynamic orthoses on gait: a retrospective control study in children with hemiplegia. Dev Med Child Neurol 50, 63-67.

Vaughan, C.L., O'Malley, M.J., 2005. Froude and the contribution of naval architecture to our understanding of bipedal locomotion. Gait Posture 21, 350-362.

Ventura, J.D., Klute, G.K., Neptune, R.R., 2011a. The effect of prosthetic ankle energy storage and return properties on muscle activity in below-knee amputee walking. Gait Posture 33, 220226.

Ventura, J.D., Klute, G.K., Neptune, R.R., 2011b. The effects of prosthetic ankle dorsiflexion and energy return on below-knee amputee leg loading. Clin Biomech 26, 298-303.

Wilken, J.M., Rodriguez, K.M., Brawner, M., Darter, B.J., 2012. Reliability and minimal detectible change values for gait kinematics and kinetics in healthy adults. Gait Posture 35, 301307.

Wu, G., Cavanagh, P.R., 1995. ISB recommendations for standardization in the reporting of kinematic data. J Biomech 28, 1257-1261.

Wu, G., Siegler, S., Allard, P., Kirtley, C., Leardini, A., Rosenbaum, D., Whittle, M., D'Lima, D.D., Cristofolini, L., Witte, H., Schmid, O., Stokes, I., 2002. ISB recommendation on definitions of joint coordinate system of various joints for the reporting of human joint motion-part I: ankle, hip, and spine. International Society of Biomechanics. J Biomech 35, 543-548.

Yang, J.F., Winter, D.A., 1984. Electromyographic amplitude normalization methods: improving their sensitivity as diagnostic tools in gait analysis. Arch Phys Med Rehabil 65, 517-521. 
Table 1. Participant demographics. $R$ indicates right side impairment, $L$ indicates left side impairment. Etiology as determined by medical assessment and record review.

\begin{tabular}{lcccccl}
\hline Participant & $\begin{array}{c}\text { Age } \\
(\mathrm{yr})\end{array}$ & $\begin{array}{c}\text { Height } \\
(\mathrm{m})\end{array}$ & $\begin{array}{c}\text { Body Mass } \\
(\mathrm{kg})\end{array}$ & $\begin{array}{c}\text { Leg Length } \\
(\mathrm{m})\end{array}$ & $\begin{array}{c}\text { Affected Side } \\
(\mathrm{R} / \mathrm{L})\end{array}$ & \multicolumn{1}{c}{ Etiology } \\
\hline 1 & 30 & 1.75 & 79.1 & 0.95 & $\mathrm{~L}$ & Tibia/fibula fracture \\
2 & 30 & 1.76 & 78.2 & 0.98 & $\mathrm{~L}$ & Tibia/fibula fracture \\
3 & 36 & 1.78 & 75.5 & 0.99 & $\mathrm{~L}$ & Talar fracture, shrapnel \\
4 & 22 & 1.64 & 80.3 & 0.91 & $\mathrm{R}$ & Shrapnel \\
5 & 27 & 1.82 & 92.9 & 0.98 & $\mathrm{R}$ & Neuropathy \\
6 & 36 & 1.95 & 82.2 & 1.11 & $\mathrm{~L}$ & Multiple fractures, shrapnel \\
7 & 26 & 1.86 & 110.4 & 1.01 & $\mathrm{R}$ & Soft tissue injury, neuropathy \\
8 & 33 & 1.77 & 90.7 & 0.98 & $\mathrm{~L}$ & Tibia fracture \\
9 & 36 & 1.76 & 83.6 & 0.93 & $\mathrm{R}$ & Multiple fractures \\
10 & 21 & 1.75 & 88.3 & 0.94 & $\mathrm{~L}$ & Shrapnel, foot fracture \\
11 & 22 & 1.97 & 99.5 & 1.07 & $\mathrm{~L}$ & Tibia/fibula fracture \\
12 & 25 & 1.69 & 91.4 & 0.90 & $\mathrm{R}$ & Soft tissue injury, neuropathy \\
13 & 40 & 1.77 & 90.8 & 0.98 & $\mathrm{R}$ & Neuropathy \\
\cline { 1 - 4 } Average & 29.54 & 1.79 & 87.92 & 0.98 & & \\
Std Dev & 6.28 & 0.09 & 9.70 & 0.06 & & \\
\hline
\end{tabular}


Table 2. Average (standard deviation) peak joint kinematics and kinetics at self-selected speed (positive values indicate dorsiflexion, knee flexion and hip flexion). The gait cycle regions during which the peaks occur are indicated as 1) first double-leg support, 2) early single-leg support, 3) late single-leg support, 4) second double-leg support, 5) early swing, and 6) late swing. Significant differences between the low and middle ( $\mathbf{\Lambda})$ and high and middle ( $\mathbf{-})$ bending axis conditions are noted. Gait measures are shaded gray for preference effects sizes $\eta^{2}>0.26$ and dark gray for $\eta^{2}>0.50$.

\begin{tabular}{|c|c|c|c|c|c|}
\hline \multirow[b]{2}{*}{ Ankle Angle } & \multirow[b]{2}{*}{ Region } & \multirow[b]{2}{*}{ Limb } & \multicolumn{3}{|c|}{ Peak Joint Kinematics $\left({ }^{\circ}\right)$} \\
\hline & & & Low & Middle & High \\
\hline \multirow[t]{2}{*}{ Plantarflexion } & 1 & PD-AFO & $-7.40(2.00)$ & $-6.33(1.89)$ & $-6.77(1.60)$ \\
\hline & & Non-PD-AFO & $-2.03(1.62)$ & $-1.25(1.98)$ & $-1.87(2.79)$ \\
\hline \multirow[t]{2}{*}{ Dorsiflexion } & 4 & PD-AFO & $5.83(1.79)$ & $5.19(1.36)$ & $4.87(1.43)$ \\
\hline & & Non-PD-AFO & $15.15(2.38)$ & $15.34(2.39)$ & $14.78(2.61)$ \\
\hline \multirow[t]{2}{*}{ Plantarflexion } & 5 & PD-AFO & $-0.68(0.54)$ & $-0.61(0.56)$ & $-0.65(0.51)$ \\
\hline & & Non-PD-AFO & $-15.31(3.83)$ & $-15.40(4.69)$ & $-16.08(5.17)$ \\
\hline \multicolumn{6}{|l|}{ Knee Angle } \\
\hline \multirow[t]{2}{*}{ Flexion } & 2 & PD-AFO & $17.34(7.45)$ & $17.46(4.19)$ & $17.85(5.97)$ \\
\hline & & Non-PD-AFO & $16.19(4.95)$ & $16.41(4.05)$ & $16.80(4.93)$ \\
\hline \multirow[t]{2}{*}{ Extension } & 3 & PD-AFO & $5.21(6.82)$ & $4.69(5.05)$ & $4.91(4.19)$ \\
\hline & & Non-PD-AFO & $1.84(2.13)$ & $3.24(2.75)$ & $2.73(3.29)$ \\
\hline \multirow[t]{2}{*}{ Flexion } & 5 & PD-AFO & $63.45(4.39)$ & $63.34(4.11)$ & $63.34(5.72)$ \\
\hline & & Non-PD-AFO & $62.76(4.01)$ & $62.86(4.10)$ & $63.26(4.78)$ \\
\hline \multicolumn{6}{|l|}{ Hip Angle } \\
\hline \multirow[t]{2}{*}{ Extension } & 4 & PD-AFO & $-3.05(4.71)$ & $-3.07(5.58)$ & $-4.48(5.13)$ \\
\hline & & Non-PD-AFO & $-5.79(4.18)$ & $-5.38(6.15)$ & $-6.36(5.97)$ \\
\hline \multirow[t]{3}{*}{ Flexion } & 6 & PD-AFO & $38.43(4.52)$ & $38.57(5.11)$ & $37.78(5.60)$ \\
\hline & & Non-PD-AFO & $34.97(3.16)$ & $35.09(3.61)$ & $34.72(4.08)$ \\
\hline & & & \multicolumn{3}{|c|}{ Peak Joint Kinetics (Nm/kg) } \\
\hline \multicolumn{6}{|l|}{ Ankle Moment } \\
\hline \multirow[t]{2}{*}{ Dorsiflexion $\boldsymbol{\Delta}$} & 1 & PD-AFO & $0.45(0.08)$ & $0.40(0.07)$ & $0.43(0.10)$ \\
\hline & & Non-PD-AFO & $0.27(0.07)$ & $0.24(0.08)$ & $0.25(0.07)$ \\
\hline \multirow[t]{2}{*}{ Plantarflexion } & 4 & PD-AFO & $-1.44(0.23)$ & $-1.50(0.17)$ & $-1.45(0.19)$ \\
\hline & & Non-PD-AFO & $-1.40(0.21)$ & $-1.43(0.19)$ & $-1.39(0.22)$ \\
\hline \multicolumn{6}{|l|}{ Knee Moment } \\
\hline \multirow[t]{2}{*}{ Flexion } & 1 & PD-AFO & $0.47(0.11)$ & $0.46(0.06)$ & $0.47(0.11)$ \\
\hline & & Non-PD-AFO & $0.62(0.09)$ & $0.60(0.09)$ & $0.61(0.08)$ \\
\hline \multirow[t]{2}{*}{ Extension } & 2 & PD-AFO & $-0.64(0.19)$ & $-0.59(0.19)$ & $-0.68(0.19)$ \\
\hline & & Non-PD-AFO & $-0.52(0.18)$ & $-0.48(0.12)$ & $-0.53(0.17)$ \\
\hline \multirow[t]{2}{*}{ Flexion } & 3 & PD-AFO & $0.38(0.14)$ & $0.43(0.18)$ & $0.35(0.09)$ \\
\hline & & Non-PD-AFO & $0.43(0.12)$ & $0.41(0.12)$ & $0.41(0.16)$ \\
\hline \multicolumn{6}{|l|}{ Hip Moment } \\
\hline \multirow[t]{2}{*}{ Extension } & 1 & PD-AFO & $-1.01(0.22)$ & $-1.00(0.13)$ & $-1.00(0.23)$ \\
\hline & & Non-PD-AFO & $-1.18(0.15)$ & $-1.17(0.10)$ & $-1.17(0.15)$ \\
\hline \multirow[t]{2}{*}{ Flexion } & 4 & PD-AFO & $0.59(0.11)$ & $0.57(0.16)$ & $0.64(0.16)$ \\
\hline & & Non-PD-AFO & $0.57(0.13)$ & $0.54(0.13)$ & $0.58(0.14)$ \\
\hline
\end{tabular}




\section{Figure Captions}

Figure 1. Passive-dynamic ankle-foot orthosis (PD-AFO). a) PD-AFO with the prescribed carbon fiber strut, b) with the proximal (high) bending axis SLS strut, c) with the central (middle) bending axis SLS strut, and d) with the distal (low) bending axis SLS strut.

Figure 2. Six regions of the gait cycle: 1) first double-leg support, 2) early single-leg support, 3) late single-leg support, 4) second double-leg support, 5) early swing, and 6) late swing.

Figure 3. Average (standard deviation bars) integrated electromyographic (iEMG) values at selfselected walking speed in the PD-AFO and non-PD-AFO limbs for the low, middle and high bending axis conditions during six regions of the gait cycle: 1) first double-leg support, 2) early single-leg support, 3) late single-leg support, 4) second double-leg support, 5) early swing, and 6) late swing. Data are presented for the gluteus medius (GMED), biceps femoris long head (BF), rectus femoris (RF), vastus medialis (VAS), medial gastrocnemius (GAS), soleus (SOL) and tibialis anterior (TA) muscles. Significant bending axis main effects $(*)$, bending axis*limb interaction effects $(\dagger)$, and low to middle bending axis comparisons $(\boldsymbol{\Delta})$ are indicated. Large effect sizes between preference and iEMG are also indicated $\left(\circ: \eta^{2}>0.26 ; \bullet: \eta^{2}>0.50\right)$.

Figure 4. Average (standard deviation bars) joint work at self-selected walking speed in the PDAFO and non-PD-AFO limbs for the low, middle and high bending axis conditions during six regions of the gait cycle: 1) first double-leg support, 2) early single-leg support, 3) late single-leg support, 4) second double-leg support, 5) early swing, and 6) late swing. Average positive and negative joint work and respective standard deviations are presented separately. Significant bending axis main effects $(*)$, low to middle bending axis comparisons $(\boldsymbol{\Delta})$ and high to middle bending axis comparisons ( $\boldsymbol{\square})$ are indicated. Large effect sizes between preference and joint work are also indicated $\left(\circ: \eta^{2}>0.26 ; \bullet: \eta^{2}>0.50\right)$.

Figure 5. Average (standard deviation bars) ground reaction force (GRF) impulses at selfselected walking speed in the PD-AFO and non-PD-AFO limbs for the low, middle and high bending axis conditions during six regions of the gait cycle: 1) first double-leg support, 2) early single-leg support, 3) late single-leg support, 4) second double-leg support, 5) early swing, and 6) late swing. Anteroposterior (A/P), vertical and mediolateral (M/L) positive and negative GRF impulses and respective standard deviations are presented separately. Significant bending axis main effects $(*)$, low to middle bending axis comparisons $(\mathbf{\Lambda})$ and high to middle bending axis comparisons ( $\mathbf{a})$ are indicated. Large effect sizes between preference and GRF impulse are also indicated $\left(\circ: \eta^{2}>0.26 ; \bullet: \eta^{2}>0.50\right)$. 
Figure 1.
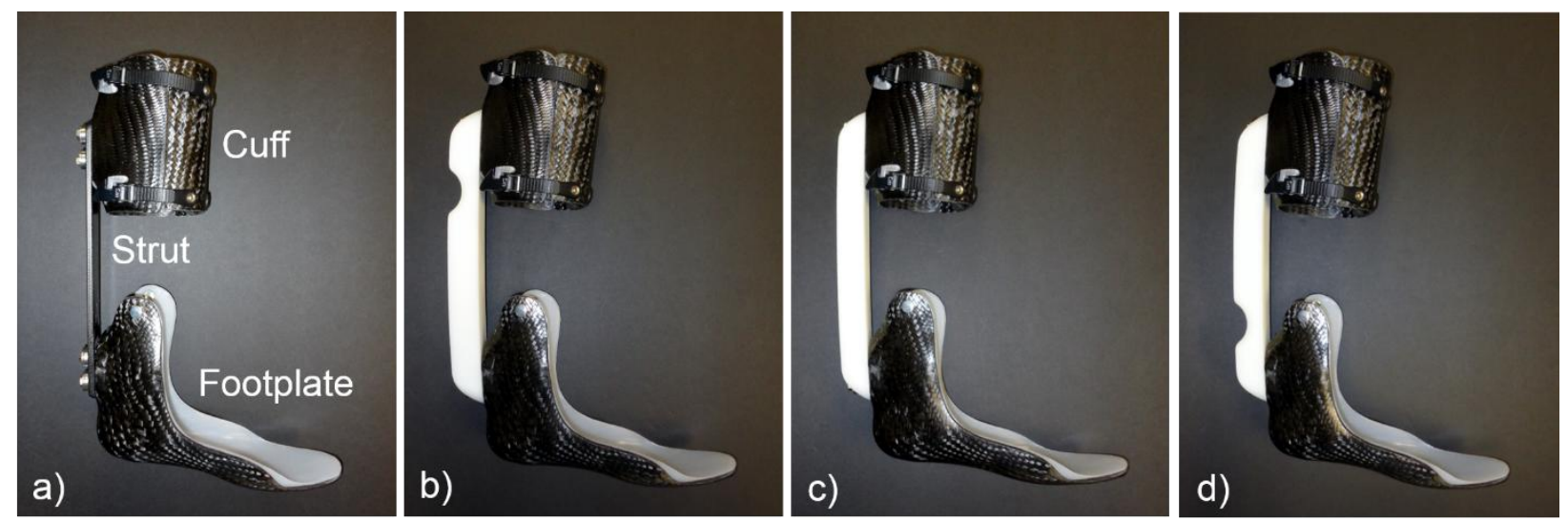
Figure 2.

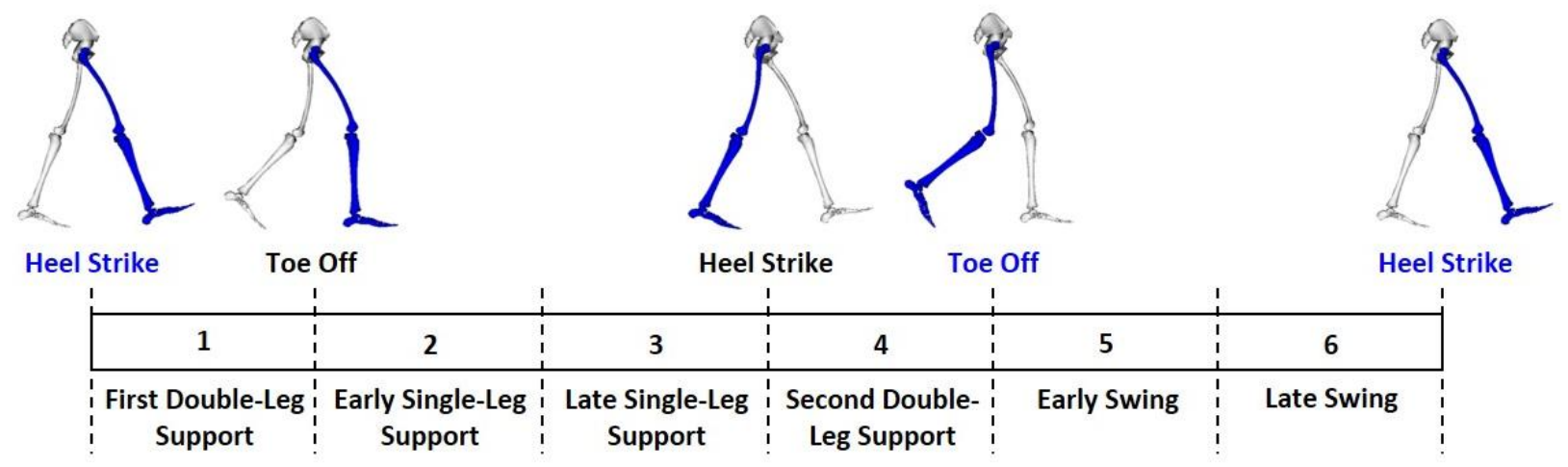


Figure 3.
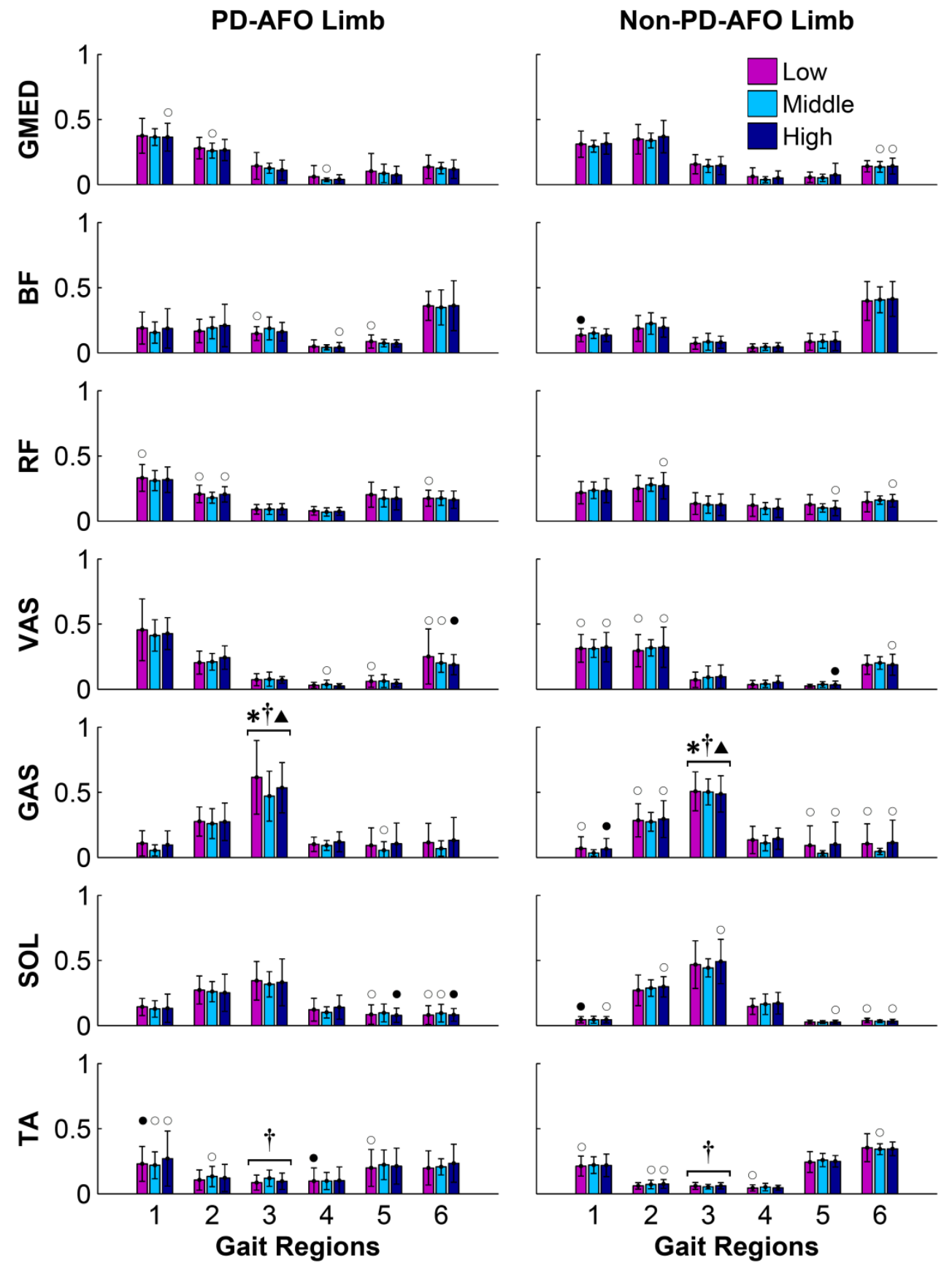
Figure 4.
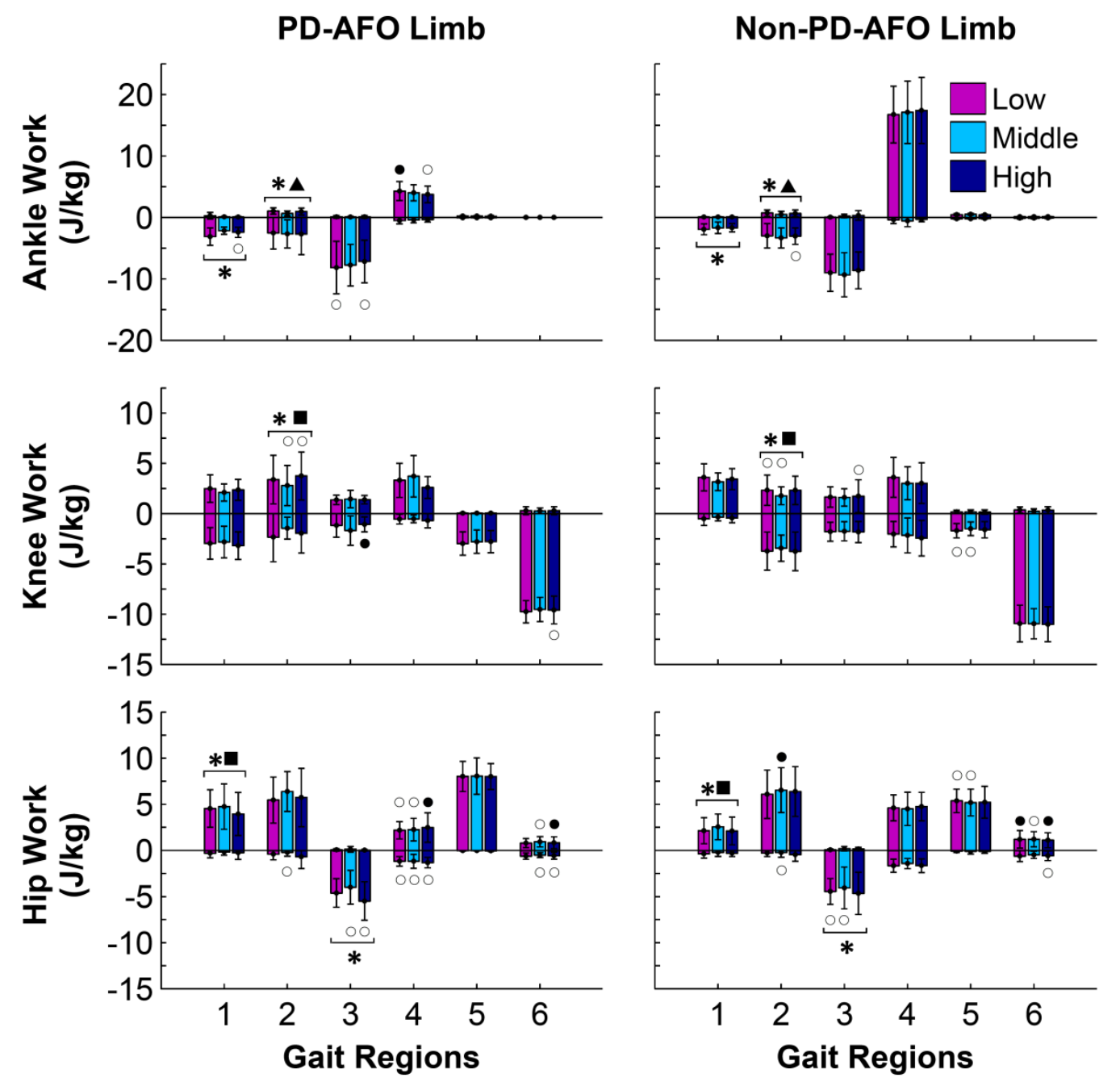
Figure 5.
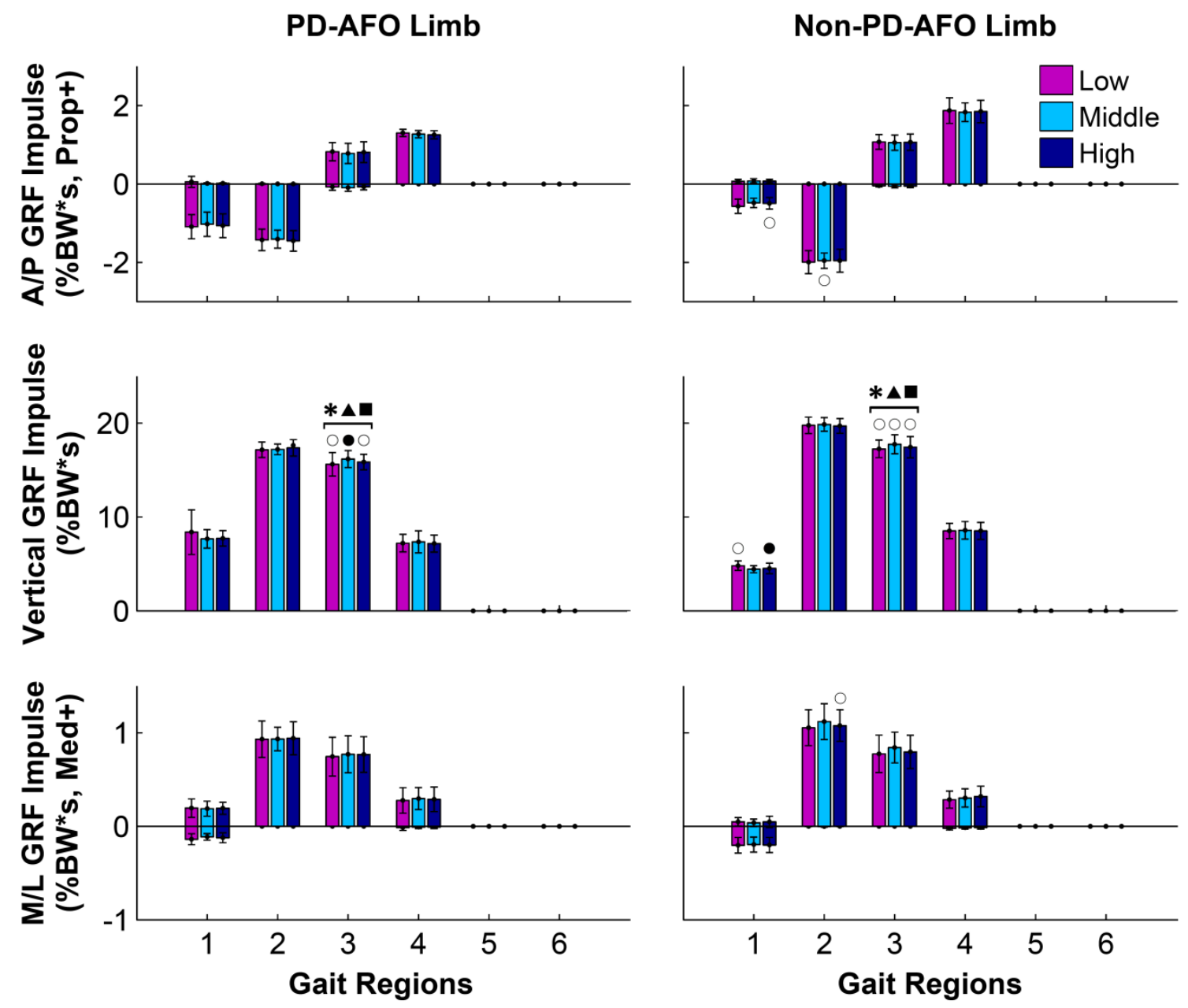